\title{
La Gobernanza frente a las estructuras burocráticas y de privatización del servicio educativo dentro de un ámbito de NGP. El caso de México
}

\author{
Quiroz Reyes, Haleyda \\ USAL, Salamanca, España \\ hale_quiroz_reyes@usal.es
}

\section{Resumen}

Vivimos frente a una ola de cambios relacionados con la aplicación de nuevas gestiones en todo el sector público, incluyendo las estructuras administrativas y prácticas del Estado, con la denominada Nueva Gestión Publica. En éste ámbito, se entiende que la gobernanza simboliza los cambios en esta forma de gobierno vinculados a los efectos de la globalización en donde el sector educativo está inmerso.

Definir el concepto gobernanza de una manera lineal, no es tarea fácil, entender este término implica, analizar diversas vertientes. Por lo que, esta investigación centra su atención en acentuar la diferencia entre la noción de ésta con la de gobierno y sus prácticas más arraigadas que controlan las estructuras burocráticas, legislaciones y regulaciones de los servicios públicos. Partiendo de esta temática global, aterrizamos teóricamente en su estructura y lineamientos aplicados en los centros escolares dentro de su organización y administración. Analizando los modelos Neoconservador y Neoliberal como bases en donde se construye esta organización entre el control por parte del primero y con la extensión del mercado por parte del segundo. Partimos de la construcción de una estructuración de factores de ambos modelos mediante un análisis cualitativo dentro de un recorrido teórico, que nos permite representar gráficamente los factores de ambos modelos, que representan los cimientos donde se implementa la NGP. Posteriormente se aplican las variables de liderazgo, actores, evaluación, autonomía y rendición de cuentas para analizar las particularidades de la gobernanza dentro de un caso de estudio: México, con sus peculiaridades propias y sus circunstancias escolares tan adversas y diversas en el ámbito de la gobernanza, dentro de una lucha contra la privatización en una disputa continua de prácticas tan arraigadas de burocracia y todo este contexto, en un ambiente que exige nuevas formas de gestión en la búsqueda continua de la calidad educativa, pero que han abierto más brechas para la exclusión.

\section{Abstract}

We live in the face of a wave of changes related to the application of new managements throughout the public sector, including the administrative structures and practices of the State, with the so-called New Public Management. In this area, it is understood that governance symbolizes changes in this form of government linked to the effects of globalization in which the education sector is immersed.

Defining the concept of governance in a linear way is not an easy task, understanding this term implies analyzing different aspects. Therefore, this research focuses its attention on accentuating the difference between the notion of the latter with that of government and its more ingrained practices that control the bureaucratic structures, legislations and regulations of public services.

Starting from this global theme, we landed theoretically in its structure and guidelines applied in schools within its organization and administration. Analyzing Neoconservative and Neoliberal models as bases where this organization is built between control by the first and the extension of the market by the second. We start with the construction of a structuring of factors of both models through a qualitative analysis within a theoretical path, which allows us to graphically represent the factors of both models, which represent the foundations where the NGP is implemented. Subsequently, the variables of leadership, actors, evaluation, autonomy and accountability are applied to analyze the particularities of governance within a case study: Mexico, with its own peculiarities and its adverse and diverse school circumstances in the field of education. governance, within a fight against privatization in a continuous dispute of practices so rooted in bureaucracy and all this context in an environment that demands new forms of management in the continuous pursuit of educational quality, but that have opened more gaps for exclusion .

Palabras clave: gobernanza, nueva gestión pública, privatización, autonomía, exclusión.

Keywords: governance, new public management, privatization, autonomy, exclusión. 


\section{INTRODUCCIÓN}

A nivel mundial se le esta concediendo cada dia mayor importancia a los temas educativos, frente a las demandas mundiales que se convierten en imperativos globales sobre cómo y quién debe administrar este servicio público ante estándares de competitividad y calidad. La figura del Estado -quién gestionaba este servicio- a mutado en diversidad de formas y bajo muy variadas circunstancias según cada país, pero con un denominador común: cada vez es más vulnerada la autonomía y la autoridad ante la economía mundial. Bajo este escenario se está priorizando la búsqueda de la eficiencia y eficacia, orientada a la formación de los recursos humanos, a la competitividad internacional de las economías nacionales, en un contexto en el que las exigencias son mayores y el Estado empieza a reducir su capacidad para mantener niveles de beneficio para la sociedad (Ball, 1998).

Hablamos de un Estado que ha modificado sus formas de administrar los servicios públicos, bajo esta dinámica al hablar de gobierno habitualmente nos referimos al partido o coaliciones políticas que controlan las estructuras estatales -el servicio público- y las prácticas estatales -elaboración de leyes y políticas, creación de regulaciones, etc.- en cualquier nación y en cualquier momento concreto (Rizvi \& Lingard, 2013), se podría considerar que el gobierno funciona en un Estado - Nación con las estructuras burocráticas del sector público. En tanto que la noción de gobernanza simboliza los cambios en esta forma de gobierno vinculados a los efectos de la globalización, con la aplicación de nuevas gestiones en todo el sector público incluyendo las estructuras administrativas y prácticas del Estado, lo que da lugar a lo que se conoce como la Nueva Gestión Pública (NGP).

La OCDE (2015) considera a la NGP primordial para desarrollar una cultura orientada hacia el rendimiento como elemento importante de la nueva forma de gobernanza en la construcción y el uso de evaluaciones comparativas y medidas que generen resultados medibles. Factores que actualmente tienen movilizados a todos los países analizando y emprendiendo acciones sobre esos resultados y sus posiciones arriba o debajo en relación con los resultados a nivel mundial.

Atendiendo a dichas exigencias globales, el rendimiento escolar ha resultado el factor detonante que estructura diversas reformas educativas, cambios en metodologías, modelos y formas de enseñar y evaluar. México no es la excepción con su Reforma Educativa gestada en el año 2012 bajo una coalición de partidos políticos que legitimaron su propuesta con las recomendaciones de organizaciones como la OCDE, BM, UNESCO entre otras y utilizando los resultados de pruebas internacionales como El Programa para la Evaluación Internacional de Alumnos (PISA) - como elemento de medición que representa la prueba más legitima y exacta de rendimiento educativo internacional y la competitividad internacional- pero sin el respaldo y la participación de profesores y comunidad escolar.

Ante este panorama surge la reflexión de repensar: ¿se ha olvidado la importancia de escuchar la voz de los actores regionales y locales de la comunidad escolar para sólo escuchar la voz global? ¿la autonomía de los centros escolares se ha perdido o en realidad algún vez se tuvo? ¿hay posibilidades de encontrar mediaciones entre las presiones burocráticas y de privatización?. Básicamente nos centramos en describir las pérdidas, los intereses, los controles y demandas que el servicio educativo esta sufriendo con la finalidad de hacernos consientes de la importancia de establecer los criterios de autonomía escolar y participación democrática donde esos intereses globales parecen encontrar un límite.

\section{LA GOBERNANZA FRENTE A LAS ESTRUCTURAS BUROCRÁTICAS DEL SERVICIO EDUCATIVO DENTRO DE UN ÁMBITO DE NGP. EL CASO DE MÉXICO}

Las estructuras de toma de decisiones están cambiando, al igual que la globalización ha transformado los terminos discursivos en los que se consideran ahora las políticas educativas vinculadas al curriculum, pedagogía y evaluación, tambien se ha transformado el modo en que se dirigen los asuntos relacionados con la gobernanza educativa y como organizar armonicamente esos elementos para gobernar las escuelas. El Estado administrativo burocrático ha sido remplazado por organizaciones policéntricas que entrañan intereses tanto públicos como privados (Rizvi y Lingard, 2013) y no bajo las necesidades y demandas que surgen de los centros escolares y la comunidad escolar -contexto local-.

La reflexión se centra en la existencia de una serie de agentes diversos y versátiles que hoy componen el servicio educativo -Estado, organizaciones internacionales, organizaciones con y sin fines de lucro, localmente profesores, personal directivo, investigadores, estudiantes, alumnos, comunidad 
escolar, etc.- que bajo ese torbellino de fenómenos globales y para su supervivencia y competitividad mundial, han emprendido esfuerzos para descentralizar los sistemas de dirección educativa que resultan primordiales para establecer la nueva forma de gobernanza y gestión pública. Es entonces cuando se desarrollan nuevas formas de organización, se presenta «un proceso de profunda transformación social» que implica la aparición de «nuevas formas de organización social, económica y política» (Tedesco, 2000, p. 11) que viven los sistemas educativos.

En este contexto en el que la toma de decisiones cada vez es mas compartida entre los diferentes actores, es indispensable dinamizar, optimizar y flexibilizar las estructuras de los servicios, mediante una visión clara de la participación de cada uno de los agentes involucrados, pensando que «la gobernanza se refiere no sólo a las estructuras formales de un sistema, sino también a cómo los gobiernos fijan prioridades e interacciones entre las partes implicadas para moldear el éxito de la política» (OCDE, 2015a, p.115).

Así, el ideal de gobernanza y de la nueva gestión pública, no se deberían separar de los propósitos educativos y tendrían que ocuparse de propiciar una participación democrática mediante una intervención local que permita atender particularidades especificas de cada una de las escuelas y la toma de decisiones en la comunidad -como características principales de la descentralización- (Rizvi y Lingard, 2013). Nos referimos a que bajo esas nuevas intervenciones de diversos actores en el servicio educativo, es indispensable que la estructura administrativa trabaje en concordancia y armonía con la estructura de toma de decisiones y objetivos, lo que se conoce como liderazgo (Kehm 2011).

En este sentido, la gobernanza eficiente tiene dos dimensiones clave, la primera se refiere a la forma en que interactúan las instituciones y los agentes implicados en el proceso de toma de decisiones, la segunda describe la «forma en que los gobiernos implementan políticas y fijan prioridades, planean y aplican nuevas políticas mediante un mezcla de presión y consulta» (OCDE, 2015a, p.37).

Esta interacción entre instituciones, actores implicados y toma de decisiones del gobierno dentro de una gobernanza conlleva una descentralización o «una delegación de funciones políticas y administrativas en los procesos de regulación de los distintos niveles de la educación» (Vega, 2011, p.251) dentro de un escenario de oportunidad activo de toma de decisiones que dentro del ámbito de los centros escolares representaría un espacio para la disertación de acuerdos en los procesos de participación local bajo una estructura de compromiso de la comunidad escolar en torno a los propósitos básicos de la educación en su contexto propio.

Para comprender la descentralización en el ámbito de la gobernanza, examinamos dos vertientes que tienen objetivos comunes como la eficiencia para mejorar la calidad, la escuela es el centro de atención, hay un enfásis en la autonomía escolar y altos grados de participación social. Pero difieren en los fundamentos y connotaciones ideologicas destacando una vertiente de lógica neoliberal que promueve que el sistema educativo funcione bajo los criterios competitivos del libre mercado, la autonomía de las escuelas representa el mecanismo que se espera eleve automáticamente la calidad de la educación dentro de criterios de competitividad y la otra vertiente centrada hacia la democratización de los proceso educativos, apuesta por una real participación social y una autonomía para responsabilizarse de lo académico y de lo administrativo (Ponton, 2003).

Pretendemos resaltar que existen dos caminos ante todos estos discursos de NGP, en términos teóricos-prácticos y no sólo de discursos globales. La descentralización y la autonomía pueden representar componentes fundamentales de la gobernanza para una participación más local y democrática que posibilitan el desarrollo de nuevos modelos de gestión escolar congruentes con la realidades, así como nuevas políticas educativas regionales que tengan que ver con contenidos curriculares, con la profesionalización de los docentes, con los procesos de evaluación bajo modelos y propuestas de participación democrática específicos y locales y no sólo para definir criterios de competitividad,

El otro camino responde a esa NGP que atiende al compromiso de garantizar la calidad del desempeño escolar, en donde esa adopción de nuevas formas de gobierno y por lo tanto de administración se orienta sobre todo a los procesos de rendición de cuentas en la búsqueda de legitimar los controles de calidad. Su elemento clave es un espacio generalizado de medición educativo a través de indicadores internacionales que exigen rendir cuentas, "dar pruebas de un uso eficiente y eficaz del dinero público» (Kehm, 2011, p. 29) y no beneficios sociales centrados en la educación de los estudiantes que sería entonces también lo que Falabella (2015) denomina como la gestión 
orientada a resultados, nutrido por la teoría de las escuelas eficaces, atendiendo a los supuestos de que la política es que los establecimientos tienen autonomía en los modos de efectuar su gestión y, a la vez, son responsables de su desempeño y deben rendir cuenta por ello.

Es un proceso que implica una doble rendición de cuentas para las escuelas, primero al mercado dando respuesta a la demanda de las familias y así captar mayor matrícula y por otra parte al Estado por el cumplimiento de estándares y metas de desempeño. Es una lógica empresarial basada en principios de competencia, riesgo, cumplimiento de metas cuantificables e incentivos ligados a resultados, mezclado con elementos de mercado competitivo sobre un bien público al servicio de todos los ciudadanos (Falabella 2015) y no un escenario de participación denocrática de gobernanza.

En este punto tendríamos que preguntarnos si la intencionalidad de la reforma educativa en México - de otorgar mayor autonomía a los centros educativos y potenciar la figura de liderazgo- esta verdaderamente en otorgar la iniciativa de la organización y administración de los centros educativos a la comunidad escolar liderada por la figura del director con una participación de la comunidad en donde se prioricen los elementos pedagógicos o sólo se trata de descentralizar para controlar de manera jerárquica. Esta duda no sólo se plantea para un país, «buena parte de los proceso de descentralización llevados a cabo en los sistemas educativos internacionales, en las dos últimas décadas, responden más a criterios de carácter económico que a otros de orientación pedagógica (la autonomía escolar)» (Vega, 2011, p.252). Hablamos de optar por una delegación de responsabilidades del Estado con modelos administrativos más flexibles, más participativos por que de otra manera están alejadas de una verdadera autonomía de gestión, que lamentablemente es la descentralización por la que camina México, como analizaremos a continuación.

El caso de México no es diferente, el esfuerzo por entrar en ese horizonte global de sistemas educativos competitivos en busca de la muy anhelada calidad educativa, que se ha convertido en una estrategia para la modernización del sistema de gestión del servicio público «cuyo diseño y propósito responde al paradigma de la Nueva Gestión Pública y la apuesta por la gestión basada en la escuela, como parte de la adopción del movimiento de escuelas eficaces» (Del Castillo, 2012, p.164) y no a un cambio centrado en la mejora de un modelo educativo pedagógicamente innovador que atienda a las necesidades específicas de un país con una diversidad muy amplia de contextos y por tanto de necesidades escolares sumamente variadas.

Ante un escenario educativo tan heterogéneo se suma que México presenta una gobernanza central compartida, ver Figura 1. Nos referimos a un poder central y regional que se traduce en un Estado responsable de diseñar y estructurar el marco legislativo, regulando sus principios, lineamientos y objetivos. En cuanto al poder regional la administración de la educación presenta diferentes grados de autonomía con apoyos de las instituciones coordinadoras, que han iniciado una delegación de responsabilidades a las autoridades regionales o locales y los centros escolares. La característica principal de este tipo de gobernanza central se basa en la importancia crucial de desarrollar una gran consistencia, capacidad y el liderazgo a nivel municipal. Los países que presentan estos modelos, se enfrentan al reto de proporcionar más autonomía con el fin de adaptarse a las necesidades locales y garantizar la coordinación efectiva entre los responsables políticos locales, regionales y nacionales (OCDE, 2015).

Figura 1. La Gobernanza en México según la OCDE.

\begin{tabular}{|c|c|c|}
\hline Tipo de gobernanza & Control de acciones y actores & Características \\
\hline & & Marco Legislativo diseña: \\
& Poder Central Regional & Principios \\
Central Compartida & Lelegación de algunas responsabilida- \\
& des locales. & Objetivos \\
& & Evaluación \\
& & Poca autonomía \\
\hline
\end{tabular}

Fuente: Elaboración propia a partir de OCDE. (2015). Política educativa en perspectiva 2015. Hacer posibles las reformas. España: Fundación Santillana. 
Este esquema permite analizar una fenómeno muy interesante de la gobernanza central en donde se genera una dinamica de delegación de responsabilidades locales -que podría significar los inicios de una autonomia para una verdadera participación local- pero así mismo se desarrollan lineamientos y medidas de evaluación y control muy estrictas para poder dar cuenta de los logros o avances de una autonomía que en suma es relativa.

Sobre este contexto la OCDE (2015) recomienda de manera global la autonomía escolar: como estrategia que conlleva profesionalizar a los líderes y exigirles que rindan cuentas, que participen en las decisiones fundamentales de sus escuelas como contratar o despedir docentes, apuesta por las estructuras de decisión que son adaptadas a sus contextos y que pueden tener un impacto positivo en el desempeño las escuelas; partiendo de esta recomendación hablaríamos de entrar en el reto de una autonomía que dirija sus proceso hacia características especificas del centro donde se aplica.

Pero la autonomía no sólo se expresa en la identidad de la institución, la toma de decisiones, la estructura y la estrategia, también se ejerce en ámbitos como el diseño de los contenidos, estrategias didácticas y pedagógicas; la administración de la institución, las políticas y el gobierno, la gestión de los recursos humanos y los servicios ofrecidos por la institución. Así, podemos distinguir cuatro tipos de autonomía para las instituciones escolares: financiera, de gestión, organizativa y didáctica (Macri, 2009).

Es indispensable detenernos a reflexionar sobre la autonomía institucional y sus elementos de tipo administrativos, de gestión y control que afectan la dirección pedagógica de la misma, estos están directamente conectados con las normas, por lo tanto es posible inferir que será baja la autonomía cuando la presión normativa (leyes, decretos, reglamentos) es alta y el resultado será la constante y abundante uniformidad escolar. Sin embargo cuando por el contrario la normativa es escasa, la autonomía es más amplia y los centros escolares serán más responsables en cuanto a eso resultados por tanto hablamos de un ambiente escolar heterogéneo y creativo con atención a soluciones locales.

Sin duda la autonomía siempre será por mucho tema de debate, por que son múltiples factores que la facilitan o la frenan y en el caso del sistema educativo mexicano en el nivel básico y media superior -constitucionalmente el rango educativo obligatorio y por tanto regulado por el estado- presenta niveles muy altos de presiones normativas que requieren tramites, procedimientos, documentación administrativa muy burocrática, etc. Dentro de este esquema, directores y profesores se centran en atender a estas exigencias administrativas coartando su creatividad para la atención y creación de innovaciones pedagógicas según sus propias necesidades.

Ante un modelo burocrático de rendición de cuentas aunado a un régimen estricto de planes y programas obligatoriamente establecidos, un currículo poco flexible y elección del profesorado desde los niveles federales, bajo este panorama los centros escolares aun se encontrarán al margen de la toma de decisiones reales, lo que se traduce en una autonomía baja. Mientras estos procedimientos altamente normativos continúen, los centros escolares no podrán ser responsables de sus acciones y estrategias emprendidas.

Es preciso, reorientar el papel los actores principales del ámbito educativo -profesores, directivos, comunidad escolar, etc.- al objeto que dejen de ser considerados como administradores e implementadores de políticas y reformas educativas con escasa intervención en los aspectos de carácter pedagógico en su formulación. Su posición debe ser de dinamizadores del proyecto educativo de los centros escolares y revalorizar esos actores en términos de implicación y participación en el diseño y propuestas curriculares y pedagógicas. La apuesta se centra en potenciar la participación local desde la base de la pirámide, un modelo de gobernanza participativo de abajo hacia arriba, para poder verdaderamente romper las estructuras burocráticas que convierten la autonomía en trámites administrativos y de control. 


\section{DISCUSIÓN Y CONCLUSIONES}

Indiscutiblemente muchas de las políticas educativas cada día son determinadas por exigencias globales como hemos analizado a los largo de este análisis, los propósitos educativos se han redefinido en términos de un conjunto más estrecho de intereses acerca del desarrollo de capital humano y el rol que debe jugar la educación para satisfacer las necesidades de la economía global y asegurar la competitividad de la economía nacional (Rizvi \& Lingard, 2013).

Pero, cualquier política o reforma educativa para su correcta implantación necesita de la participación de los actores implicados en su aplicación, que estos agentes hagan suyas dichas políticas para que estas se puedan desarrollar de forma óptima y elevar el rendimiento de las mismas. Si en una política educativa los profesores no se sienten implicados, la capacidad técnica de implementación no tendrá un impacto positivo y esta es la realidad de la reforma sobre la calidad en México, los actores primordiales del proceso educativo, no fueron participes de esta reforma y están regidos aún por un modelo que privilegia la exigencia de resultados, lo que genera mayor presión en los centros escolares por rendir cuentas de resultados.

La gestión escolar en México, no se encuentra equilibrada si de vertientes de aplicación hablamos, está dirigida mayormente a los aspectos administrativos con la formulación y exigencias de formatos de rendimiento de cuentas, así como una excesiva normativa para el seguimiento de los espacios de participación y en la elaboración del proyecto escolar -que son diseñados en las cúpulas más altas y los profesores simplemente siguen esos lineamientos-, estas condiciones no permiten una verdadera construcción colaborativa donde participen todos los actores del proceso educativo entorno a su creatividad y sus necesidades. El desenlace es una gestión escolar que no presenta aún un rango de autonomía escolar abierto, esta bajo un rango de operatividad para presentar resultados.

Cuando hablamos del proceso de rendición de cuentas es indispensable girar las miradas otra vez a los actores de liderazgo en los centros escolares -los directivos-, en los cuales recae esta responsabilidad y de los cuales depende en gran parte la innovación y el manejo diferente de este proceso de gobernanza de autonomía de gestión. Lamentablemente en México los lideres escolares presentan severas presiones que imposibilitan el cambio o generan la continua realización de practicas de administración tradicionales por que se enfrentan continuamente a procedimientos burocráticos de control de rendición de cuentas y no hay espacios ni oportunidades de practicar una liderazgo pedagógico en un entorno de autonomía relativa.

Específicamente en México la autonomía institucional parece necesaria para flexibilizar el sistema y para mejorar sus resultados, pero se enfrenta con importantes dificultades tanto en el plano económico como social. Esta doble realidad supone un desafío importante a la hora de diseñar y aplicar políticas públicas, ya que la viabilidad de las mismas puede verse seriamente amenazada en el contexto presente (Egido et al., 2000). Al hablar del cuestiones de carácter económico, tanto la escasez de los recursos asignados a los centros como el control administrativo que de ellos se realiza, limitan la capacidad de decisión institucional a nivel local, estos son factores son determinantes para limitar una verdadera autonomía de gestión estando en manos de otros niveles de la administración y por lo tanto limitan la gobernanza local.

La ansiada descentralización del sistema hasta el nivel de los propios centros de enseñanza produce en ocasiones problemas de diferente índole para su aplicación, debido a prácticas administrativas tradicionales arraigadas durante muchos años provenientes de un sistema educativo eminentemente burocrático. Ejemplo de este obstáculo para la adecuada puesta en práctica de la autonomía escolar, es que aún el gobierno federal es quien opera y dispone de elementos trascendentales para la gestión escolar; es decir, aún es quien conserva la autoridad normativa, técnica y pedagógica, para el funcionamiento de la educación obligatoria en México. Es quien diseña, implementa, autoriza y evalúa los programas de estudio, los libros de texto, el calendario escolar, los cuadernillos de seguimiento de los consejos técnicos escolares -espacios de participación-, la evaluación del aprendizaje y de los docentes, etc. Mientras estos elementos sigan siendo responsabilidad de la autoridad educativa central, la autonomía estará limitada para el diseño de proyectos educativos específicos en los centros escolares. El resultado es que, los centros escolares se han limitado a rendir cuentas según la normatividad y bajo este esquema el Estado no ha disminuido su poder, sino que ha cambiado los modos de ejercer el control a distancia, es decir los centros educativos solo tienen una libertad normada. 
Esto nos lleva a concluir que las escuelas bajo una gobernanza frente a estructuras burocráticas y en un ambiente de exigencias globales, sólo tienen una autonomía de gestión relativa presentan niveles muy altos de presiones normativas que requieren tramites, procedimientos, documentación administrativa muy burocrática, un régimen estricto de planes y programas obligatoriamente establecidos, un currículo poco flexible y elección del profesorado que se hace desde los niveles federales. Todo esto responde meramente a una meta central que exige rendición de resultados bajo exámenes internacionales e instancias evaluadoras y no a necesidades específicas de los centros a nivel local. Mientras estos procedimientos continúen siendo altamente normativos, no podrán ser responsables de sus acciones y estrategias emprendidas y por ende aún se encuentran al margen de la toma de decisiones reales; lo que se traduce en una autonomía limitada.

México también realiza una doble rendición de cuentas, no sólo tienen que detallar cuentas al mercado, dando respuesta a la demanda de las familias, también tienen que dar cuentas al Estado por el cumplimiento de estándares y metas de desempeño. Bajo un proceso burocrático que ha representado otra debilidad institucional en cuanto a organización, operación y autonomía de las escuelas y que a lo largo del tiempo se ha podido evidenciar, sumado a un panorama de desigualdad dentro de las circunstancias de cada centro escolar. México no se han distinguido por llevar con eficiencia sus procesos, al contrario lamentablemente se han distinguido por una burocracia que restringe la autonomía de gestión en los centros educativos y conduce sólo a una estrategia de rendición de cuentas y no una oportunidad de gobernanza local. 


\section{REFERENCIAS BIBLIOGRÁFICAS}

Ball, S. (1998), Big policies, small world. Comparative Education, (34) 2, p. 119-30.

Del Castillo, A. (2012). Las Políticas Educativas en México desde una perspectiva de política pública: gobernabilidad y gobernanza. Magis, Revista Internacional de Investigación en Educación , 4 (9), 637-652.

Egido, I. (2015). El liderazgo escolar como ámbito de la política educativa supranacional. Madrid: Bordón. Revista de Pedagogía, 67(1), pp.71-84.

Falabella, A. (2015) El mercado escolar en Chile y el surgimiento de la Nueva Gestión Pública: El tejido de la política entre la dictadura neoliberal y los gobiernos de la centro izquierda (1979 a 2009). Educaçao \& Sociedade , 36 (132), 1-21.

Kehm, B. (2011). La gobernanza en la enseñanza superior. Sus significados y su relevancia en una época de cambios. (M. L. Urrutia, Trans.) Barcelona: Educación Universitaria OCTAEDRO- ICE.

Macri, M. (2009). Descentralización educativa y autonomía institucional ¿Constituye la descentralización un proceso abierto hacia la autonomía de las escuelas públicas de Buenos Aires? OEl- Revista Iberoamericana de Educación .

OCDE. (2015). Política educativa en perspectiva 2015. Hacer posibles las reformas. España: Fundación Santillana.

OCDE. (2015a). Estudios Económicos De La OCDE En México, Visión General 2015. OCDE.

Ponton, C. (2003). La descentralización de los sistemas educativos. Revista Mexicana de Investigación Educativa , 8 (18), 283-290.

Rizvi , F., \& Lingard, B. (2013). Politicas educativas en un mundo globalizado. (1a ed.) Madrid: Morata.

Tedesco, J. (2000) Educar en la sociedad del conocimiento. Buenos Aires: FCE. Vega, L. (2011). Gobernanza y políticas de formación inicial de profesores en la Europa mediterránea. Valencia: Tirant lo Blanch. 